\title{
A drastic complex atheromatous aorta A case report
}

\section{Ateromasia aortica complicata. Un caso clinico}

\author{
Ivano Bonadei1, Enrico Vizzardi1, Antonio D’Aloia1, Edoardo Sciatti2, Edoardo Cervi², \\ Stefano Bonardelli2, Marco Metra1, Stefano Maria Giulini2
}

\begin{abstract}
A drastic complex atheromatous aorta. I. Bonadei, E. Vizzardi, A. D'Aloia, E. Sciatti, E. Cervi, S. Bonardelli, M. Metra, S.M. Giulini.

Aortic atherosclerosis is the most common disease of the aorta. More than 50\% of the plaques thicker than 4 $\mathrm{mm}$ are located along the descending aorta. The complex morphology of the plaque, such as ulceration or the presence of thrombi, is associated with increased embolic risk.
\end{abstract}

The increasing use of transesophageal echocardiogram has enhanced the recognition of aortic atheromas. We describe a case of a male patient with complex atherosclerotic disease involving the coronary vessels and descending aortic tract with some embolic complications.

Keywords: aortic stenosis, thrombus, atherosclerosis.

Monaldi Arch Chest Dis 2013; 80: 45-47.

\footnotetext{
1 Department of medical and surgical, Radiological Sciences and Public Health specialties, Section of Cardiovascular Diseases, University of Brescia.

2 Department of Medical and Surgical Sciences, Surgical Clinic, University of Brescia.
}

Corresponding author: Dr. Enrico Vizzardi; Department of medical and surgical, Radiological Sciences and Public Health specialties, Section of Cardiovascular Diseases, University of Brescia, Italy; Tel. + 39 303995679; E-mail address: enrico.vizzardi@tin.it

\section{Case report}

We describe a case of a 70-year-old male who came to the emergency room due to dyspnea and cough despite antibiotic therapy for about one week. Chest X-ray showed moderate right pleural effusion associated with signs of pulmonary stasis and calcifications of the aorta. His history included hypertension (treated with beta-blockers) smoking and alcohol abuse. The patient was transferred to our Department and was started on therapy with boluses of intravenous diuretics. An ARB was introduced for better blood pressure control and progressive improvement of dyspnea. However, during hospitalization, the patient had an onset of angina with evidence of ST depression and onset of negative T waves in V5-V6 on electrocardiogram performed. He was submitted to trans thoracic echocardiogram which showed a dilated left ventricle with akinesia of the anterior and inferior mid-basal segments and basal septal walls determining moderate global systolic dysfunction (ejection fraction 35\%-40\%) and evidence of aortic stenosis. There was a modest increase in the markers of myocardial necrosis. So we began double antiplatelet therapy and intravenous nitrates added to $20 \mathrm{mg}$ of simvastatin and it was planned the coronary angiography. Meanwhile, for the detection of marked bradycardia and some episodes of asystole on telemetry (Figure 1) the patient was transferred to our intensive care unit and it was implanted temporary pacemaker. Coronary angiography detected moderate coronary artery disease: moderate stenosis of the proximal left anterior descending coronary and to ostial of right coronary artery (RCA); before a revascularization procedure we planned a study of myocardial viability in the territory of the RCA. Returning from the hemodynamic room the patient reported the onset of temporary phosphenes in the right eye. He was evaluated by a neurologist who suggested a retinal periprocedural microembolism. Another echocardiogram was performed which confirmed what had been revealed earlier. However moderate aortic calcified stenosis was noted (delta P max 35-40 mm Hg, AVA 1.5 - 1.6 $\mathrm{cm} 2$ ), subsequently confirmed by transesophageal echocardiograpy (TEE) (Figure 2). It also showed the presence of a marked atherosclerotic aortic wall associated with moving parts floating in the descending aorta that were not present in the aortic arch (Figure 3). The patient was treated with heparin, enhanced antihypertensive therapy and simvastatin was replaced with $40 \mathrm{mg}$ of atorvastatin. Due to the onset of inflammation, the assessment for possible revascularization and implantation of pacemaker was delayed. During the subsequent hospitalization, the patient began complaining of a stabbing pain and cramping of the left lower limb, associated with absence of peripheral pulses and hypothermia. A vascular surgeon was contacted who raised the suspicion of acute lower limb thrombotic and embolic ischemia. The patient underwent thromboembolectomy using a Fogarty catheter followed by resumption of anticoagulation.

After a few hours we noted the emergence of an acute ischemia of the right leg. The surgeon was again contacted and he gave an indication for angiography with evidence of obstruction at the common femoral artery. So the patient underwent thromboembolectomy at the femoral bifurcation with profundoplasty with a bovine patch and right superficial femoral 
artery ligation. Upon return from the operating room, the patient showed no signs of bilateral flow and he resumed heparin therapy. In the following hours he suddenly became comatose; angio-computed tomography was performed showing the evidence of right internal carotid artery thrombosis and hyperacute ischemia in the territory of the middle cerebral artery (recanalized by the oars of the Willis polygon). Given the poor prognosis and the framework itself there was no indication for intensive treatment.

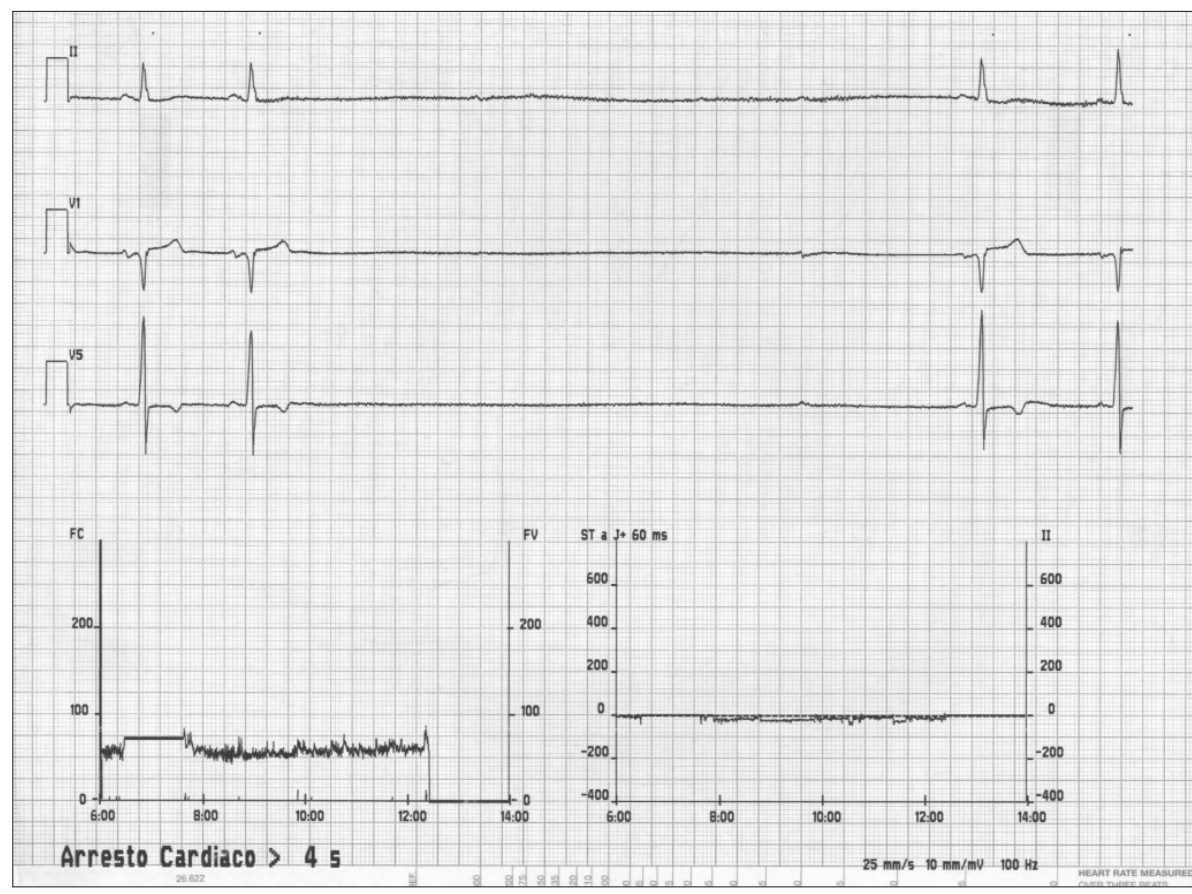

Figure 1. - The electrocardiogram shows a pause $>4 \mathrm{sec}$.

\section{Discussion}

Aortic atherosclerosis (AA) is the most common disease of the aorta. The aortic plaques located along the ascending aorta and aortic arch are the most frequent causes of ischemic embolic stroke. Recently it has been demonstrated that complex descending aorta plaques should be considered as a new source of stroke [1]. More than $50 \%$ of the plaques thicker than $4 \mathrm{~mm}$ are located along the descending aorta. The plaques may be calcified, ulcerated, soft or with superimposed thrombi. A simple classification divides the atheromatous plaque into simple and complex or stable and unstable. The complex morphology of

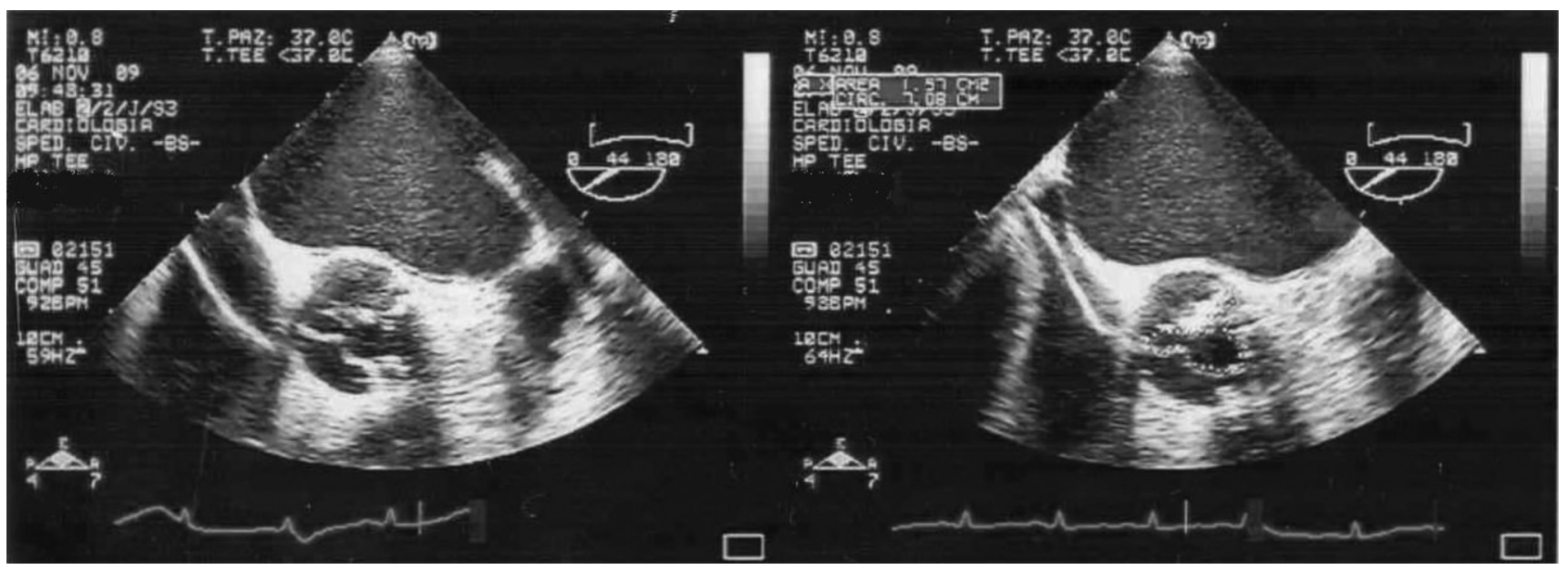

Figure 2. - Aortic stenosis at Echo TEE.

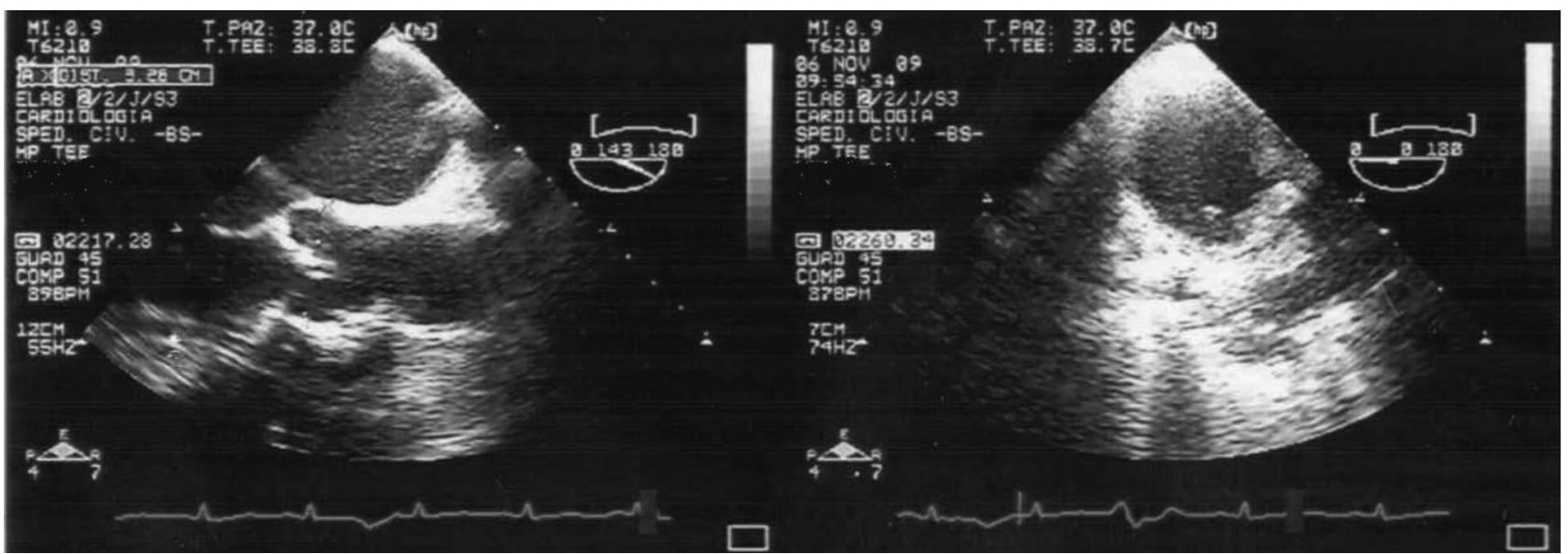

Figure 3. - Presence of marked atherosclerotic aortic wall throughout the aortic's course associated with trombus floating in the descending aorta. 
the plaque, such as ulceration or the presence of thrombi, is associated with increased embolic risk, while the presence of calcification indicates a lower risk. Plaque morphology is very dynamic with frequent formations and resolution of mobile components [2]. In the literature, the embolic risk associated with AA was significant if placque thickness was greater than $4 \mathrm{~mm}$ and if it was complex (e.g. moving parts increase the risk of stroke and death by 17-fold). AA is often associated with atherosclerosis in other vascular beds such as the carotid artery and coronary arteries [3]; an association with severe calcific aortic stenosis and mitral annular calcification has also been observed. These conditions are predisposed to embolisms and therefore aortic atheroma may be a marker of other disease states causing stroke $[4,5]$. In particular, the simultaneous presence of atherosclerotic aortic and aortic valve stenosis has been increasingly recognized [6]; At our institution we noted that $63 \%$ of patients with aortic valve stenosis had severe aortic atheromatous plaque $(>4$ $\mathrm{mm}$ ) compared to $35 \%$ with mild aortic stenosis.

It has been estimated that the relative risk for stroke or peripheral embolism in patients with severe AA has quadrupled and is even more than 12 in patients with mobile atheromas and fluctuating components; its prevalence among patients presenting with ischemic stroke is similar to that of atrial fibrillation and atheromatous carotid (20\%). Recurrent stroke is common in patients with aortic arch atheroma with a thickness of more than $4 \mathrm{~mm}$ or mobile components, particularly in the categories of high-risk patients such as the elderly, smokers, hypertensives and diabetics. Patients who have severe and complex atheroma are at increased risk of recurrence (14\% per year) and could therefore require a more aggressive secondary prevention strategy. The increasing use of TEE has enhanced the recognition of aortic atheromas. In patients with cryptogenetic stroke, it is reasonable to verify the presence of a severe and complex aortic atheroma by the use of TEE [7]. Although magnetic resonance and computed tomography investigations are emerging non-invasive diagnoses for characterization of aortic atheromas, TEE is still a high-level investigation [8, 9].

AA treatment has not yet been well defined and there are still conflicting results regarding the use of anticoagulant. Statins, by their mechanisms of plaque stabilization, appear to be a promising approach $[10,11]$. Surgical treatment should be considered for patients with abdominal aortic or popliteal artery aneurysms and downstream atheroembolism. There are case reports of atheroemboli in patients worsening after being given warfarin or heparin. For this reason, some institutions are reluctant to prescribe these drugs for patients with atheroemboli or thromboemboli from aortic plaque. However, the incidence of this com- plication is quite low. Anticoagulation should probably be stopped if a patient develops an atheroembolism. Unstable aortic plaques may develop superimposed thrombi, easily seen as mobile elements on TEE. Therefore, it is possible that anticoagulation with warfarin might prevent embolic events in these patients. Currently, the combination of dual antiplatelet therapy or anticoagulation with warfarin are used. Which of these two treatments are more effective will be evident after the end of the study ARCH (Aortic Arch Related Cerebral Hazard trial) [12].

In the described case it should be noted that neither the introduction of high-dose statin therapy nor the use of dual antiplatelet and anticoagulant therapy changed the prognosis of the patient.

\section{References}

1. Harloff A, Simon J, Brendecke S, et al. Complex plaques in the proximal descending aorta: an underestimated embolic source of stroke. Stroke 2010 Jun; 41(6): 1145-50.

2. Casella G, Greco C, Perugini E et al. Atheromatosis of thoracic aorta and risk of stroke. G Ital Cardiol 2006; 7 (5): 309-316

3. Mahabadi AA, Bamberg F, Toepker M, et al. Association of aortic valve calcification to the presence, extent, and composition of coronary artery plaque burden: from the Rule Out Myocardial Infarction using Computer Assisted Tomography (ROMICAT) trial. Am Heart J 2009 Oct; 158(4): 562-8.

4. ThenappanT, Jafar AR, Assad M. Aortic atheromas: current concepts and controversies-a review of the literature. Echocardiography 2008; 25: 198-207

5. Elias-Smale SE, Odink AE, Wieberdink RG, et al. Carotid Aortic arch and coronary calcification are related to history of stroke: the Rotterdam Study. Atherosclerosis 2010 Oct; 212(2): 656-60

6. Osranek M, Pilip A, Patel P et al. Amounta of aortic atherosclerosis in patients with aortic stenosis as determined by transesophageal achocardiography. Am J Cardiol 2009; 103: 713-717

7. Jones EF, Kalman JM, Calafiore P, et al. Proximal aortic atheroma. An independent risk factor for cerebral ischemia. Stroke 1995; 26: 218-224

8. Choukroun EM, Labrousse LM, Madonna FP, et al. Mobile thrombus of the thoracic aorta: diagnosis and treatment in 9 cases. Ann Vasc Surg 2002 Nov; 16(6): 714-22. Epub 2002 Nov 7.

9. Frazin LJ, Glowney JW. Mobile ascending aortic atheroma diagnosed by transesophageal echocardiography as source of peripheral vascular embolism. $J$ Am Soc Echocardiogr 2009 Aug; 22(8): 972.e1-4.

10. Tunick PA, Nayar AC, Goodkin GM, et al. Effect of treatment on the incidence of stroke and other emboli in 519 patients with severe thoracic aortic plaque. Am J Cardiol 2002 Dec 15; 90(12): 1320-5.

11. Yonemura A, Momiyama Y, Fayad ZA, et al. Effect of lipid-lowering therapy with atorvastatin on atherosclerotic aortic plaques: a 2-year follow-up by noninvasive MRI. Eur J Cardiovasc Prev Rehabil 2009 Apr; 16(2): 222-8

12. http://clinicaltrials.gov/ct2/show/NCT00235248 